INPLASY

PROTOCOL

To cite: He et al. The effect of isotretinoin in the treatment of acne vulgaris on insulin resistance: a systematic review and meta-analysis. Inplasy protocol 202190065. doi: 10.37766/inplasy2021.9.0065

Received: 21 September 2021

Published: 21 September 2021

Corresponding author:

Li Changqiang

731977853@qq.com

Author Affiliation: SOUTHWEST MEDICAL UNIVERSITY.

Support: No.

Review Stage at time of this submission: The review has not yet started.

Conflicts of interest: None declared.

\section{The effect of isotretinoin in the treatment of acne vulgaris on insulin resistance: a systematic review and meta-analysis}

$\mathrm{He}, \mathrm{Q}^{1}$; Li, C².

Review question / Objective: P: acne vulgaris; I: isotretinoin/ 13 cis Retinoic Acid; O: Insulin Resistance(HOMA-IR); S: Nonrandomized controlled trial.

Eligibility criteria: 1 Inclusion criteria1.1 Research objects All research objects should meet the diagnosis of acne vulgaris in the 2019 "Chinese Acne Treatment Guidelines". Exclude breastfeeding or pregnant women, exclude those who are allergic to drug ingredients, and exclude patients who have used anti-inflammatory drugs, antibacterial drugs, and lightsensitive drugs within one month.1.2 Intervention measures Patients are treated with isotretinoin, the dose and course of treatment are not limited.1.3 Outcome indicators HOMA-IR1.4 Research type After searching for randomized controlled trials, there are too few literatures to conduct Meta analysis. Therefore, the scope was expanded to include nonrandomized controlled trials.2 Exclusion criteria (1) Duplicate publications; (2) Systematic reviews, meta-analysis, and review; (3) Literatures with incomplete data.

INPLASY registration number: This protocol was registered with the International Platform of Registered Systematic Review and Meta-Analysis Protocols (INPLASY) on 21 September 2021 and was last updated on 21 September 2021 (registration number INPLASY202190065).

\section{INTRODUCTION}

Review question / Objective: P: acne vulgaris; I: isotretinoin/13 cis Retinoic Acid; O: Insulin Resistance(HOMA-IR); S: Nonrandomized controlled trial.
Condition being studied: Acne is a chronic inflammatory skin disease that occurs during adolescence and mainly affects the sebaceous glands of the hair follicles on the face. Its pathogenesis has not yet been fully elucidated. Hormone-induced sebaceous gland excessive secretion of 
lipids, abnormal hair follicle sebaceous duct keratosis, Propionibacterium acnes and other hair follicle microbe proliferation, inflammation and immune response are related to it in the genetic background. Studies have shown that the onset of acne vulgaris is related to insulin resistance. Isotretinoin is a conventionally used oral tretinoin drug at home and abroad. It is often used as the first choice for the treatment of acne vulgaris. It is usually $0.25-0.5 \mathrm{mg} / \mathrm{kg} \cdot \mathrm{d}$ as the starting dose, and then it can be gradually used according to the patient's tolerance and efficacy. Adjust the dose, severe nodular cystic acne can be gradually increased to $0.5 \sim 1.0 \mathrm{mg} / \mathrm{kg} / \mathrm{d}$. Over the years, there have been many conflicting reports on whether the effect of Isotretinoin in the treatment of acne vulgaris on insulin resistance is statistically significant. In July 2019, Tsung-Yu Tsai published "effects of isotretinoin on glucose metabolism in patients with acne: A systematic review and meta-analysis", stating that isotretinoin treatment will not affect insulin resistance. However, from July 2019 to August 2021, some nonrandomized controlled trials on the effect of isotretinoin in the treatment of acne vulgaris on insulin resistance have been added in the world.

\section{METHODS}

Participant or population: Acne vulgaris.

Intervention: Isotretinoin/13 cis Retinoic Acid.

Comparator: Healthy people.

Study designs to be included: Nonrandomized controlled trial.

Eligibility criteria: 1 Inclusion criteria1.1 Research objects All research objects should meet the diagnosis of acne vulgaris in the 2019 "Chinese Acne Treatment Guidelines". Exclude breastfeeding or pregnant women, exclude those who are allergic to drug ingredients, and exclude patients who have used anti-inflammatory drugs, antibacterial drugs, and lightsensitive drugs within one month.1.2
Intervention measures Patients are treated with isotretinoin, the dose and course of treatment are not limited.1.3 Outcome indicators HOMA-IR1.4 Research type After searching for randomized controlled trials, there are too few literatures to conduct Meta analysis. Therefore, the scope was expanded to include non-randomized controlled trials.2 Exclusion criteria (1) Duplicate publications; (2) Systematic reviews, meta-analysis, and review; (3) Literatures with incomplete data.

Information sources: PubMed, Embase, Web of Science, Cochrane Library, SinoMed, CNKI, VIP, WanFang.

Main outcome(s): HOMA-IR.

Quality assessment / Risk of bias analysis: Cochrane risk bias assessment tool.

Strategy of data synthesis: No heterogeneity chooses fixed-effects to merge data. If there is heterogeneity, first examine the source of the heterogeneity, and choose a reasonable way to merge the data. For example, if the source of the heterogeneity is statistical heterogeneity, you can choose to merge the data with random effects.

Subgroup analysis: No subgroups.

Sensitivity analysis: After deleting the data of any one of the documents, is the result of the merged data of the remaining documents greatly deviated from the original.

Country(ies) involved: China.

Keywords: Isotretinoin; Acne vulgaris; Insulin resistance; Systematic review; Meta analysis.

Contributions of each author:

Author 1 - HE QINGQING.

Author 2 - LI CHANGQIANG. 